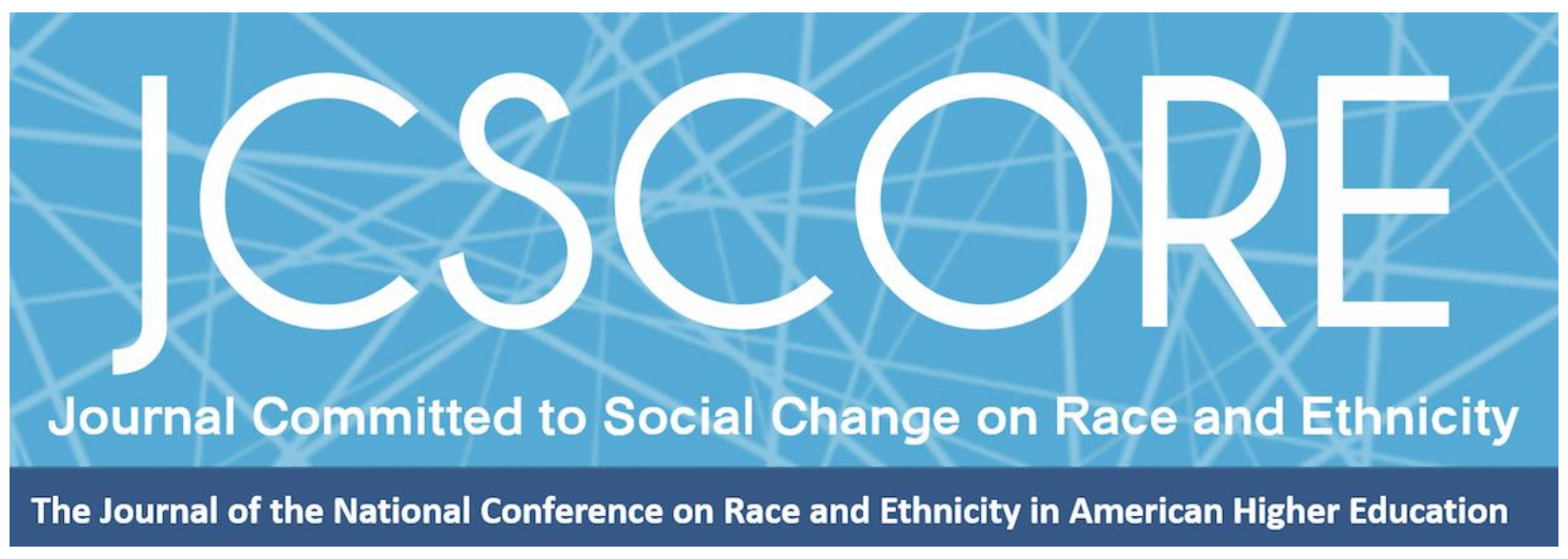

\title{
STRATEGIES FOR PRACTICING SELF-CARE FROM RACIAL BATTLE FATIGUE
}

Stephen John Quaye

The Ohio State University

Shamika N. Karikari

Miami University 1

Courtney Rashad Allen1

Wilson Kwamogi Okello

University of North Carolina Wilmington

Kiaya Demere Carter

DHL Express

Journal Committed to Social Change on Race and Ethnicity

Volume 5, Issue 2 | 2019

Copyright (C) 2019 Board of Regents of The University of Oklahoma on behalf of the Southwest Center for Human Relations Studies.

Permission of the Publisher is required for resale or distribution and for all derivative works, including compilations and translations. Quoting small sections of text is allowed as long as there is appropriate attribution. 
Journal Committed to Social Change on Race and Ethnicity | Volume 5, Issue 2 | 2019

Strategies for Practicing Self-Care from Racial Battle Fatigue

\author{
Stephen John Quaye \\ The Ohio State University \\ Shamika N. Karikari \\ Miami University 1 \\ Courtney Rashad Allen1 \\ Wilson Kwamogi Okello \\ University of North Carolina Wilmington \\ Kiaya Demere Carter \\ DHL Express
}

\begin{abstract}
Racial battle fatigue is the exhaustion that People of Color feel from repeated exposure to racism, as well as its negative impact on their emotional, physiological, and psychological health and wellbeing. Although People of Color have engaged in resistance and resilience in the midst of racism, it still takes a toll on their bodies. In this article, we focus specifically on Black student affairs educators, given the racial battle fatigue they navigate working in a helping profession where they are often expected to prioritize students' needs above their own. Using a narrative methodological approach, we centered the stories of 35 Black student affairs educators across various institutions to identify the strategies they used to practice self-care in the midst of racial battle fatigue, including unplugging from the people and places that caused them harm, building community with other Black educators, caring for their bodies, finding safe spaces, and using counseling. We offer implications for practice for Black student affairs educators and those working to support them in navigating racial battle fatigue.
\end{abstract}

Racism is an everyday, ordinary experience with which People of Color must contend. Given the history of slavery and white supremacy, Black people, in particular, navigate a particular form of racism rooted in anti-Blackness (Dumas \& ross, 2016), which means Black people working at predominantly white institutions must navigate repeated racism and a particular hatred directed toward them as Black people (Delgado \& Stefancic, 2011; Smith, Hung, \& Franklin, 2011). This daily exposure to anti-Black 
Journal Committed to Social Change on Race and Ethnicity | Volume 5, Issue 2 | 2019

racism can lead to racial battle fatigue, which describes the ways Black people are exhausted and suffer physically, emotionally, and mentally (Smith, Allen, \& Danley, 2007) from the cumulative effects of racism.

In order to survive and thrive in the face of racism, Black people must develop strategies for taking care of themselves and coping in the midst of racism. Although Black people are not responsible for the racism they experience, prioritizing their own self-care is essential for healthy wellbeing. History records subtle and deliberate efforts to dispossess, disembody, and erase the possibility of Black lives (Alexander, 2004), and thus, survival has meant equally committed counter-warfare in the struggle for Black bodies to secure and retain personhood. Black people often take up the resilient words of Harlem Renaissance poet McKay (1919): "We'll face the murderous, cowardly pack, pressed to the wall, dying, but fighting back" (p. 1)! Fighting back involves the various strategies Black people employ to dream, imagine, and build communities that enable them to thrive in the midst of racism (Love, 2019). As a counterclaim to assailment, the act of creating is always, first, an effort to preserve one's existence, or what we discuss in this paper as self-care.

The purpose of this narrative study was to explore the specific strategies Black student affairs educators used to practice self-care and heal from racial battle fatigue. We see self-care and healing as different processes; the latter is long-term and more sustainable. However, both are needed to navigate racism. In this paper, we focus on self-care strategies. We chose student affairs educators for this study given the time they invest in working with college students and how they often prioritize students' needs over their own care (Miller, 2016). We wanted to see how Black student affairs 
Journal Committed to Social Change on Race and Ethnicity | Volume 5, Issue 2 | 2019

educators worked to center their own needs in practicing self-care. Racism can impact Black student affairs educators in particular ways since they work with students who are also navigating racism themselves. Hearing how participants navigated this tension is important for researchers and educators invested in supporting Black student affairs educators.

\section{Literature Review}

Prior to discussing the methodology and methods we employed for this study, we situate our study in the extant literature. Three bodies of literature informed our work: emotional labor, racial battle fatigue, and grit and self-care.

\section{Emotional Labor}

Stemming from Pierce's (1996) work on women in work settings, emotional labor describes how women manage or control their emotions to protect men's feelings so that men do not dismiss them in the workplace. This emotional management is an extra or added layer of labor in which men do not have to participate, resulting in women not being able to spend that time elsewhere. Researchers have extended this work to Populations of Color, noting how People of Color engage in emotional labor often to the benefit of white people in those spaces (Evans \& Moore, 2015). By managing their emotions, not expressing their anger as a means to protect white people's feelings, People of Color also engage in emotional labor (DiAngelo, 2011).

This emotional labor means they cannot devote that energy toward self-care strategies, which might enable them to preserve their dignity in historically white spaces:

Participation in white institutional spaces requires particular forms of emotional labor and management of emotions from people of color, resulting from the stark contradiction between their racialized experiences in these institutions, on the 
Journal Committed to Social Change on Race and Ethnicity | Volume 5, Issue 2 | 2019

one hand, and the dominant discourse that minimizes and delegitimizes their experiences on the other hand. (Evans and Moore, 2015, p. 441)

Because white supremacy means that white people frame Black people as overly emotional, aggressive, and dangerous (Smith, Mustaffa, Jones, Curry, \& Allen, 2016), emotional labor means Black people are often constantly managing their emotions so that white people do not accuse them of acting in the ways white racial frames imagine them to be. This constant emotional management is exhausting; for example, when Black people point out racism, white people then frame them as being combative, overly sensitive, and not able to handle themselves in professional settings (DiAngelo, 2011; Smith et al., 2016). White people's anger is legitimate, but it is irrational or unbecoming for Black people to respond with similar anger (Evans \& Moore, 2015). Because United States society, and thus workplaces, are constructed as fair and equitable, when Black people point out disparities, white people can easily frame them as troublemakers (Evans \& Moore, 2015). Since white people can express emotion and still be seen as professional, they can then offer perfectly logical explanations for why they are not racist in the face of Black people pointing out racism. White people's denials make Black people look "crazy" or illogical for pointing out racial inequities in the workplace (Evans \& Moore, 2015).

\section{Racial Battle Fatigue}

The emotional management of white people's feelings, as well as not expressing the full range of their emotions can exacerbate racial battle fatigue. Critical race theorists have explicated how racism is an everyday, ordinary experience with which People of Color navigate (Delgado \& Stefancic, 2012). The ordinariness of racism does not mean one should ignore its debilitating effects. Although racism may be less overt in 
Journal Committed to Social Change on Race and Ethnicity | Volume 5, Issue 2 | 2019

present-day society, often existing through more subtle, seemingly innocuous forms (i.e., microaggressions) (Sue, 2010), repeated exposure to racism can lead to racial battle fatigue, that is, the sheer exhaustion People of Color feel. Although this tiredness is a real phenomenon, even more important are the negative health implications from racial battle fatigue, including higher blood pressure, anxiety, and stress (Husband, 2016; Smith, 2004, 2009a, 2009b; Smith, Yosso, \& Solórzano, 2006).

Racial battle fatigue is also gendered. For example, given the intersection of sexism and racism for Black cisgender women, their experience of racial battle fatigue is different from that of Black cisgender men (Corbin, Smith, \& Garcia, 2018). For example, having to fend off stereotypes of the angry Black woman can exacerbate the aforementioned health consequences (Harris-Perry, 2011). Using the metaphor of the crooked room, Harris-Perry conveyed how Black women are often misrepresented because of the ways white people, Black men, and non-Black People of Color fail to see them. Seeing Black women through the lens of stereotypes that they are either aggressive, angry people or overly strong caretakers who save everyone both misrepresent Black women and fail to allow them space to be human (Corbin et al., 2018; Harris-Perry, 2011). Relatedly, Black men navigate Black misandry, or antiBlackness, which views them as inhuman. As such, white people often surveil Black men and view them as dangerous, thugs, gangsters, and not belonging within higher education institutions (Smith et al., 2007). A particular hatred toward Black bodies exists, and misogynoir and Black misandry help explain how genderism intersects with racism to exacerbate feelings of racial battle fatigue among Black people (BeauboeufLafontant, 2008; Smith et al., 2007). 
Journal Committed to Social Change on Race and Ethnicity | Volume 5, Issue 2 | 2019

\section{Grit and Self-Care}

Literature on people navigating strenuous environments tends to lead toward survival strategies laced in positive psychology (Dweck, 2006; Schreiner, 2010; Duckworth \& Duckworth, 2016). The common thread in and throughout this research is that generally, people want to thrive, desiring to live in ways that bring happiness and fulfillment (Duckworth \& Duckworth, 2016). Positive psychologists submit that the achievement of these markers of happiness is determined by one's ability to point their thoughts in the direction of positive outcomes. Some researchers have shown that concepts, such as grit, which is defined as the perseverance and passion that one has for long-term success, predicts a wide range of positive outcomes (e.g., Duckworth \& Duckworth, 2016). Gritty people are distinguished by their propensity to stick with a task, maintain their effort and interest "over years despite failure, adversity, and plateaus in progress" (Duckworth, Peterson, Matthews, \& Kelly, 2007, p. 1088).

The caveat with concepts like grit is that researchers employing these concepts sometimes fail to explicitly examine the role that race and racism play in prolonged perseverance and sustained efforts. Grit has particular consequences for people who hold racially minoritized identities, such as Black people in the present study. McGee and Stovall (2015) note how "the psychological and emotional energy required to manage stress in academic and social contexts as well as systemic and everyday racism can be overwhelming and taxing" (p. 493). Grit sometimes ignores the fundamental significance of history and the sociopolitical strain of existing in a white supremacist United States context. 
Journal Committed to Social Change on Race and Ethnicity | Volume 5, Issue 2 | 2019

Self-care describes the processes people use to cope in the midst of experiencing pain, trauma, violence, or in the case of the present study, racism (Miller, 2016). Student affairs, as a helping profession, often attracts people who are committed to prioritizing students' needs over their own (Miller, 2016). Self-care, in many ways, has become a buzzword used in student affairs, which often ignores the nuances of taking care of oneself. For instance, Squire and Nicolazzo (2019) underscored how self-care is often rhetoric-a vacuous concept that fails to consider systems of oppression and the role of power involved in self-care.

\section{Summary and Connection to Present Study}

The aforementioned literature review illustrates the ways Black people navigate racial battle fatigue, as well as the emotional labor in which they engage in white spaces. This means Black student affairs educators are not immune to the effects of racism and racial battle fatigue, which are exacerbated because they are working with Students of Color, who are also experiencing trauma from racism (Corbin et al., 2018; Smith et al., 2016). "Black faculty may think that ameliorating RBF [racial battle fatigue] is a hopeless endeavor, as RBF symptoms can reduce their sense of control" (Arnold, Crawford, \& Khalifa, 2016, p. 911). Black student affairs educators' self-care is a form of resilience and resistance, whereby they create and succeed even in the midst of racism. Resilience does not mean ignoring their racial battle fatigue, but instead, finding ways to mitigate the severity of it. Even so, because "resilience is no substitute for optimal mental, physical and emotional wellness" (Arnold et al., 2016, p. 911), developing healthy strategies for navigating racial battle fatigue is important. The present study sought to understand how Black student affairs educators work to do so. 
Journal Committed to Social Change on Race and Ethnicity | Volume 5, Issue 2 | 2019

\section{Methodology and Methods}

We employed narrative inquiry for this study. Narrative inquiry centers stories from participants (Clandinin \& Connelly, 2000) in order to make sense of their lived experiences. Clandinin and Rosiek (2007) noted: "narrative inquiry is not only on individuals' experience but also on the social, cultural, and institutional narratives within which individuals' experiences are constituted, shaped, expressed, and enacted" ( $p$. 42). To account for these larger narratives, we also paid attention to how participants' experiences were situated within larger systems of oppression, such as racism and sexism. We centered participants' narratives about racism and the stories they told that challenged dominant ways of knowing and systems of oppression.

\section{Positionality}

Patel (2016) calls on researchers to adopt a different way of explicating their positionality in the research process through asking themselves three questions: why me/us, why this particular study, and why now? We share our positionality as researchers using this format.

Why me/us? A research team comprised of one faculty member, three doctoral students, and one master's student undertook this study. All five research team members identify as Black; more specifically, two identify as Black cisgender, heterosexual, non-disabled women (i.e., Shamika and Kiaya), and three as Black, cisgender, heterosexual, non-disabled men (i.e., Stephen, Courtney, and Wilson). Even though we are not monolithic as Black people, our shared racial identity with each other and participants fostered rapport with participants and each other. Because we understand the significance of racism, we did not minimize participants' experiences 
Journal Committed to Social Change on Race and Ethnicity | Volume 5, Issue 2 | 2019

with it, and we worked to push ourselves when we believed shared knowledge existed. For example, rather than merely saying, "Yes," when a participant said, "You know what I mean?" we asked them to explain so that we did not rely upon a seemingly shared understanding of Blackness and race.

Why this particular study? Since racism is an ordinary experience, Black people often simply accept it as a fact of life in order to cope and survive in the midst of racism (Kendi, 2019). We engaged in this particular study as a way to not just accept racism and that Black people need to just survive. We wanted to understand, specifically, the strategies Black people use to self-care, and possibly even heal from racism. Our belief was that by centering stories of self-care, other Black student affairs educators might learn methods they can employ and not feel so isolated in their own experiences.

Why now? As we talked with Black student affairs educators on our campuses and at conferences and reflected on our own experiences, we felt a heaviness around fatigue from racism. We also sensed a feeling of hopelessness and despair. These acute feelings prompted our desire to study Black student affairs educators as they worked to navigate racial battle fatigue. Seeing the negative health effects of racial battle fatigue, we felt compelled to engage in this study now in order to learn intervention strategies for healthier ways of being in the midst of racism.

\section{Data Collection and Analysis}

We used social media (i.e., Facebook, Twitter, Instagram) to send out a call for participants who identified as Black and were currently employed as student affairs educators at colleges or universities across the country. Following this call, within 24 
Journal Committed to Social Change on Race and Ethnicity | Volume 5, Issue 2 | 2019

hours, 104 participants expressed interest in the study. Knowing we could not adequately interview everyone who expressed interest, we broke down the study into multiple phases. For the first phase, we invited the first 50 people who completed a form to participate, and ultimately, 35 people responded to our invitation.

Participants for the study were 35 Black student affairs educators who work at various colleges and universities in the United States. Participants hold various social identities beyond their Black racial identity (i.e., gender, sexual orientation, class). 24 participants identify as cisgender women, 10 as cisgender men, and one as non-binary. 27 participants identify as heterosexual, three as queer, two as gay, and one as pansexual. Participants also describe their class identity as working class (9), middleclass (18), lower-middle class (6), and upper-middle class (2). Although we are not sure, we do wonder if our identifying as cisgender, heterosexual people is reflective of the largely cisgender, heterosexual sample. To protect participants' identities, we did not list their institutions. The majority of participants were fairly new to their current position; specifically, 20 had been in the role a year or less, and 13 were in the role between two to five years. Most (i.e., 15) participants had worked between 5-10 years in higher education, with eight working 2-5 years, five working a year or less, and seven working 10 or more years. Participants ranged in entry-, mid-, and senior-level positions with seven in coordinator positions, eight in assistant director/dean titles, four as associate directors, 11 as directors, and one occupying a faculty role. These numbers do not add up neatly to 35 , as some people held multiple titles. See Table 1 for the aforementioned participant demographics and further details about each participant (names are pseudonyms chosen by each participant). 
Table 1. Participant Demographics

\begin{tabular}{|c|c|c|c|c|c|c|c|c|c|}
\hline Pseudonym & Role/Department & $\begin{array}{l}\text { Time } \\
\text { in } \\
\text { Role }\end{array}$ & $\begin{array}{l}\text { Worked } \\
\text { Full- } \\
\text { Time in } \\
\text { Higher } \\
\text { Ed. }\end{array}$ & $\begin{array}{l}\text { Educational } \\
\text { Background }\end{array}$ & Race & Gender & $\begin{array}{l}\text { Sexual } \\
\text { Orientation }\end{array}$ & Ability & SES \\
\hline Anna May & $\begin{array}{l}\text { Residential programs } \\
\text { and services }\end{array}$ & 1 year & 1 year & $\begin{array}{l}\text { Bachelors, } \\
\text { working on } \\
\text { masters }\end{array}$ & $\begin{array}{l}\text { Black/African } \\
\text { American }\end{array}$ & Woman & Queer & $\begin{array}{l}\text { Able- } \\
\text { bodied }\end{array}$ & $\begin{array}{l}\text { Working } \\
\text { class }\end{array}$ \\
\hline Becky & Global management & $\begin{array}{l}\text { a few } \\
\text { months }\end{array}$ & $\begin{array}{l}\text { a few } \\
\text { months }\end{array}$ & $\begin{array}{l}\text { bachelors and } \\
\text { masters }\end{array}$ & Black & Woman & Pansexual & $\begin{array}{l}\text { No } \\
\text { disabilities }\end{array}$ & $\begin{array}{l}\text { Working } \\
\text { class }\end{array}$ \\
\hline bell & $\begin{array}{l}\text { Coordinator for } \\
\text { student assistance }\end{array}$ & 1 year & 1 year & $\begin{array}{l}\text { bachelors and } \\
\text { masters }\end{array}$ & Black & Woman & Heterosexual & $\begin{array}{l}\text { Able- } \\
\text { bodied }\end{array}$ & $\begin{array}{l}\text { Middle } \\
\text { class }\end{array}$ \\
\hline $\begin{array}{l}\text { Black } \\
\text { Panther }\end{array}$ & $\begin{array}{l}\text { Center coordinator } \\
\text { and adjunct } \\
\text { instructor }\end{array}$ & 8 years & $\begin{array}{l}13 \\
\text { years }\end{array}$ & $\begin{array}{l}\text { bachelors, } \\
\text { masters, and } \\
\text { Ph.D. }\end{array}$ & Black & Man & Heterosexual & $\begin{array}{l}\text { Able- } \\
\text { bodied }\end{array}$ & $\begin{array}{l}\text { Working } \\
\text { class }\end{array}$ \\
\hline Colin & $\begin{array}{l}\text { Assistant dean of } \\
\text { students and director } \\
\text { of intercultural center }\end{array}$ & 1 year & 6 years & $\begin{array}{l}\text { bachelors, } \\
\text { masters, and } \\
\text { Ph.D. }\end{array}$ & Black & Man & & $\begin{array}{l}\text { Able- } \\
\text { bodied }\end{array}$ & $\begin{array}{l}\text { Middle } \\
\text { class }\end{array}$ \\
\hline Denise & $\begin{array}{l}\text { Assistant director of } \\
\text { diversity and } \\
\text { inclusion fellows / } \\
\text { advisor }\end{array}$ & 1 year & 5 years & $\begin{array}{l}\text { masters in } \\
\text { higher } \\
\text { education }\end{array}$ & $\begin{array}{l}\text { African } \\
\text { American }\end{array}$ & Female & Heterosexual & $\begin{array}{l}\text { Able- } \\
\text { bodied, } \\
\text { epilepsy }\end{array}$ & $\begin{array}{l}\text { Middle } \\
\text { class }\end{array}$ \\
\hline Destiny & $\begin{array}{l}\text { Assistant dean of } \\
\text { student life and } \\
\text { director of } \\
\text { multicultural } \\
\text { international student } \\
\text { services }\end{array}$ & 1 year & 8 years & $\begin{array}{l}\text { bachelors, } \\
\text { masters, and } \\
\text { Ph.D. }\end{array}$ & Black & Woman & Queer & $\begin{array}{l}\text { Temporarily } \\
\text { able-bodied }\end{array}$ & $\begin{array}{l}\text { Upper } \\
\text { middle } \\
\text { class }\end{array}$ \\
\hline $\mathrm{El}$ & $\begin{array}{l}\text { Assistant director of } \\
\text { student life }\end{array}$ & 4 years & $\begin{array}{l}10 \\
\text { years }\end{array}$ & $\begin{array}{l}\text { bachelors, } \\
\text { masters, and } \\
\text { Ph.D. }\end{array}$ & $\begin{array}{l}\text { Black/African } \\
\text { American }\end{array}$ & Male & & $\begin{array}{l}\text { Able- } \\
\text { bodied }\end{array}$ & $\begin{array}{l}\text { Middle } \\
\text { class }\end{array}$ \\
\hline Elaine & $\begin{array}{l}\text { Residential } \\
\text { coordinator / interim }\end{array}$ & $\begin{array}{l}2 \\
\text { months }\end{array}$ & 2 years & masters & Black & Female & Heterosexual & $\begin{array}{l}\text { Able- } \\
\text { bodied }\end{array}$ & $\begin{array}{l}\text { Middle } \\
\text { class }\end{array}$ \\
\hline
\end{tabular}




\begin{tabular}{|c|c|c|c|c|c|c|c|c|c|}
\hline Pseudonym & Role/Department & $\begin{array}{l}\text { Time } \\
\text { in } \\
\text { Role }\end{array}$ & $\begin{array}{l}\text { Worked } \\
\text { Full- } \\
\text { Time in } \\
\text { Higher } \\
\text { Ed. }\end{array}$ & $\begin{array}{l}\text { Educational } \\
\text { Background }\end{array}$ & Race & Gender & $\begin{array}{l}\text { Sexual } \\
\text { Orientation }\end{array}$ & Ability & SES \\
\hline & $\begin{array}{l}\text { director of housing } \\
\text { and residence life }\end{array}$ & & & & & & & & \\
\hline Eugene & $\begin{array}{l}\text { Assistant director of } \\
\text { fraternity and sorority } \\
\text { life }\end{array}$ & 3 years & 5 years & masters & Black & Cisgender & Heterosexual & $\begin{array}{l}\text { Able- } \\
\text { bodied }\end{array}$ & $\begin{array}{l}\text { Lower- } \\
\text { middle }\end{array}$ \\
\hline Imani & $\begin{array}{l}\text { Director of the office } \\
\text { of diversity affairs }\end{array}$ & $\begin{array}{l}11 \\
\text { months }\end{array}$ & $\begin{array}{l}15 \\
\text { years }\end{array}$ & $\begin{array}{l}\text { bachelors, } \\
\text { masters, and } \\
\text { Ph.D. }\end{array}$ & $\begin{array}{l}\text { Black/African } \\
\text { American }\end{array}$ & Female & Heterosexual & $\begin{array}{l}\text { Able- } \\
\text { bodied }\end{array}$ & $\begin{array}{l}\text { lower- } \\
\text { middle } \\
\text { class }\end{array}$ \\
\hline Jackie & $\begin{array}{l}\text { Coordinator for men } \\
\text { of color succession } \\
\text { issues }\end{array}$ & $\begin{array}{l}5 \\
\text { months }\end{array}$ & 2 years & $\begin{array}{l}\text { bachelors and } \\
\text { masters }\end{array}$ & $\begin{array}{l}\text { Black/African } \\
\text { American }\end{array}$ & Female & Heterosexual & $\begin{array}{l}\text { Not } \\
\text { disabled }\end{array}$ & $\begin{array}{l}\text { working } \\
\text { class }\end{array}$ \\
\hline Jamal & $\begin{array}{l}\text { Resident director in } \\
\text { office of residence } \\
\text { life }\end{array}$ & $\begin{array}{l}36 \\
\text { hours }\end{array}$ & 1 year & $\begin{array}{l}\text { bachelors and } \\
\text { masters }\end{array}$ & Black & Male & Heterosexual & $\begin{array}{l}\text { Able- } \\
\text { bodied }\end{array}$ & $\begin{array}{l}\text { Middle } \\
\text { class }\end{array}$ \\
\hline Jeff & $\begin{array}{l}\text { Assistant professor } \\
\text { of educational } \\
\text { leadership }\end{array}$ & 1 year & 6 years & $\begin{array}{l}\text { bachelors, } \\
\text { masters, and } \\
\text { Ph.D. }\end{array}$ & $\begin{array}{l}\text { Black, very } \\
\text { black }\end{array}$ & Male & Gay & Able & $\begin{array}{l}\text { Upper } \\
\text { middle } \\
\text { class }\end{array}$ \\
\hline $\begin{array}{l}\text { Jessie } \\
\text { Thomas }\end{array}$ & $\begin{array}{l}\text { Mentorship program } \\
\text { coordinator and } \\
\text { assistant director for } \\
\text { career advising }\end{array}$ & $\begin{array}{l}8 \\
\text { months }\end{array}$ & $\begin{array}{l}12 \\
\text { years }\end{array}$ & Not answered & Black & Female & Heterosexual & Very able & $\begin{array}{l}\text { Middle } \\
\text { class }\end{array}$ \\
\hline Joy & $\begin{array}{l}\text { Assistant athletic } \\
\text { director / director } \\
\text { compliance }\end{array}$ & $\begin{array}{l}7 \\
\text { weeks }\end{array}$ & 3 years & $\begin{array}{l}\text { bachelors and } \\
\text { masters }\end{array}$ & Black & Female & Heterosexual & $\begin{array}{l}\text { Fully } \\
\text { capable }\end{array}$ & $\begin{array}{l}\text { Middle } \\
\text { class }\end{array}$ \\
\hline Kate & $\begin{array}{l}\text { Associate director of } \\
\text { masters of arts and } \\
\text { teaching programs }\end{array}$ & 2 years & 7 years & $\begin{array}{l}\text { masters and } \\
\text { bachelors }\end{array}$ & Black & Female & Heterosexual & $\begin{array}{l}\text { Able- } \\
\text { bodied }\end{array}$ & $\begin{array}{l}\text { Middle } \\
\text { class }\end{array}$ \\
\hline Kathy & $\begin{array}{l}\text { Resident education } \\
\text { coordinator }\end{array}$ & 1 year & 8 years & $\begin{array}{l}\text { bachelors and } \\
\text { masters }\end{array}$ & Black & Female & Heterosexual & Very able & $\begin{array}{l}\text { Working } \\
\text { class }\end{array}$ \\
\hline
\end{tabular}




\begin{tabular}{|c|c|c|c|c|c|c|c|c|c|}
\hline Pseudonym & Role/Department & $\begin{array}{l}\text { Time } \\
\text { in } \\
\text { Role }\end{array}$ & $\begin{array}{l}\text { Worked } \\
\text { Full- } \\
\text { Time in } \\
\text { Higher } \\
\text { Ed. }\end{array}$ & $\begin{array}{l}\text { Educational } \\
\text { Background }\end{array}$ & Race & Gender & $\begin{array}{l}\text { Sexual } \\
\text { Orientation }\end{array}$ & Ability & SES \\
\hline Lo & $\begin{array}{l}\text { Associate director of } \\
\text { alumni relations }\end{array}$ & $\begin{array}{l}4.5 \\
\text { years }\end{array}$ & $\begin{array}{l}12 \\
\text { years }\end{array}$ & $\begin{array}{l}\text { bachelors and } \\
\text { masters }\end{array}$ & $\begin{array}{l}\text { Black/African } \\
\text { American }\end{array}$ & Cisgender & Heterosexual & Full abilities & $\begin{array}{l}\text { Lower } \\
\text { middle } \\
\text { class }\end{array}$ \\
\hline Maria & Academic advisor & $\begin{array}{l}4 \\
\text { months }\end{array}$ & 7 years & $\begin{array}{l}\text { bachelors and } \\
\text { masters }\end{array}$ & $\begin{array}{l}\text { Black/African } \\
\text { American }\end{array}$ & Woman & Straight & $\begin{array}{l}\text { Able- } \\
\text { bodied }\end{array}$ & $\begin{array}{l}\text { Middle } \\
\text { class }\end{array}$ \\
\hline Marian & $\begin{array}{l}\text { Associate director of } \\
\text { student life }\end{array}$ & 3 years & 7 years & $\begin{array}{l}\text { bachelors and } \\
\text { masters }\end{array}$ & Black & Woman & Heterosexual & Capable & $\begin{array}{l}\text { Lower } \\
\text { middle } \\
\text { class }\end{array}$ \\
\hline Maurice & Residence director & new & 1 year & $\begin{array}{l}\text { bachelors and } \\
\text { masters }\end{array}$ & Black & Man & Gay & $\begin{array}{l}\text { Temporarily } \\
\text { able-bodied }\end{array}$ & $\begin{array}{l}\text { Working } \\
\text { class }\end{array}$ \\
\hline Mercy & $\begin{array}{l}\text { Assistant director } \\
\text { office of residence } \\
\text { life and housing }\end{array}$ & 5 years & 7 years & $\begin{array}{l}\text { bachelors and } \\
\text { masters and } \\
\text { Ed.D. }\end{array}$ & Black & Female & Heterosexual & $\begin{array}{l}\text { Anxiety but } \\
\text { no other } \\
\text { issues }\end{array}$ & $\begin{array}{l}\text { Middle } \\
\text { class }\end{array}$ \\
\hline Nathan & Admissions & 1 year & 8 years & $\begin{array}{l}\text { bachelors and } \\
\text { masters }\end{array}$ & Black & $\begin{array}{l}\text { Non- } \\
\text { binary, } \\
\text { queer }\end{array}$ & queer & $\begin{array}{l}\text { Able- } \\
\text { bodied }\end{array}$ & $\begin{array}{l}\text { Working } \\
\text { poor }\end{array}$ \\
\hline Nicole & $\begin{array}{l}\text { Director of residential } \\
\text { communities }\end{array}$ & $\begin{array}{l}2.5 \\
\text { years }\end{array}$ & $\begin{array}{l}14 \\
\text { years }\end{array}$ & $\begin{array}{l}\text { bachelors and } \\
\text { masters }\end{array}$ & $\begin{array}{l}\text { Black/African } \\
\text { American }\end{array}$ & $\begin{array}{l}\text { Cisgender } \\
\text { female }\end{array}$ & heterosexual & able-bodied & $\begin{array}{l}\text { working } \\
\text { class }\end{array}$ \\
\hline Randle & $\begin{array}{l}\text { Director of student } \\
\text { affairs case } \\
\text { management }\end{array}$ & 1 year & $\begin{array}{l}24 \\
\text { years }\end{array}$ & $\begin{array}{l}\text { bachelors and } \\
\text { masters }\end{array}$ & $\begin{array}{l}\text { African } \\
\text { American }\end{array}$ & Male & Straight & $\begin{array}{l}\text { Largely } \\
\text { able }\end{array}$ & $\begin{array}{l}\text { Lower } \\
\text { middle } \\
\text { class }\end{array}$ \\
\hline Ray & $\begin{array}{l}\text { Residence hall } \\
\text { coordinator in } \\
\text { department of } \\
\text { residence life and } \\
\text { housing }\end{array}$ & 2 years & 2 years & $\begin{array}{l}\text { bachelors and } \\
\text { masters }\end{array}$ & $\begin{array}{l}\text { African } \\
\text { American }\end{array}$ & Woman & heterosexual & $\begin{array}{l}\text { Able- } \\
\text { bodied }\end{array}$ & $\begin{array}{l}\text { Middle } \\
\text { class }\end{array}$ \\
\hline Rick Ross & $\begin{array}{l}\text { Sorority senior } \\
\text { coordinator }\end{array}$ & 4 years & 8 years & $\begin{array}{l}\text { bachelors and } \\
\text { masters }\end{array}$ & Black & Male & heterosexual & $\begin{array}{l}\text { No } \\
\text { disabilities }\end{array}$ & $\begin{array}{l}\text { Middle } \\
\text { class }\end{array}$ \\
\hline Sara & $\begin{array}{l}\text { Director of } \\
\text { enrollment services } \\
\text { center }\end{array}$ & 2 years & $\begin{array}{l}12 \\
\text { years }\end{array}$ & $\begin{array}{l}\text { masters and } \\
\text { bachelors }\end{array}$ & Black & Female & heterosexual & able-bodied & $\begin{array}{l}\text { middle } \\
\text { class }\end{array}$ \\
\hline
\end{tabular}




\begin{tabular}{|c|c|c|c|c|c|c|c|c|c|}
\hline Pseudonym & Role/Department & $\begin{array}{l}\text { Time } \\
\text { in } \\
\text { Role }\end{array}$ & $\begin{array}{l}\text { Worked } \\
\text { Full- } \\
\text { Time in } \\
\text { Higher } \\
\text { Ed. }\end{array}$ & $\begin{array}{l}\text { Educational } \\
\text { Background }\end{array}$ & Race & Gender & $\begin{array}{l}\text { Sexual } \\
\text { Orientation }\end{array}$ & Ability & SES \\
\hline Sedgwick & $\begin{array}{l}\text { Director of fraternity } \\
\text { and sorority life and } \\
\text { diversity initiatives / } \\
\text { residential director / } \\
\text { first-year experience } \\
\text { faculty member }\end{array}$ & $\begin{array}{l}1.5 \\
\text { years }\end{array}$ & 4 years & $\begin{array}{l}\text { bachelors and } \\
\text { masters }\end{array}$ & $\begin{array}{l}\text { Black/African } \\
\text { American }\end{array}$ & Male & straight & able-bodied & $\begin{array}{l}\text { lower } \\
\text { SES }\end{array}$ \\
\hline Shehers & $\begin{array}{l}\text { Community director } \\
\text { in department of } \\
\text { residential education } \\
\text { and housing services }\end{array}$ & 4 years & 9 years & $\begin{array}{l}\text { bachelors and } \\
\text { masters }\end{array}$ & $\begin{array}{l}\text { Black, super } \\
\text { black }\end{array}$ & Woman & heterosexual & $\begin{array}{l}\text { completely } \\
\text { physical } \\
\text { and bipolar }\end{array}$ & $\begin{array}{l}\text { lower- } \\
\text { middle }\end{array}$ \\
\hline Sheryl & $\begin{array}{l}\text { Director of } \\
\text { admissions }\end{array}$ & $\begin{array}{l}1 \\
\text { month }\end{array}$ & $\begin{array}{l}10 \\
\text { years }\end{array}$ & $\begin{array}{l}\text { bachelors and } \\
\text { masters }\end{array}$ & $\begin{array}{l}\text { Biracial, } \\
\text { black, and } \\
\text { white but } \\
\text { identify as } \\
\text { Black }\end{array}$ & Female & heterosexual & able & $\begin{array}{l}\text { middle } \\
\text { class }\end{array}$ \\
\hline $\mathrm{TC}$ & $\begin{array}{l}\text { Coordinator of } \\
\text { student integrity }\end{array}$ & $\begin{array}{l}1.5 \\
\text { years }\end{array}$ & 5 years & $\begin{array}{l}\text { bachelors and } \\
\text { masters }\end{array}$ & Black & Female & heterosexual & able & $\begin{array}{l}\text { middle } \\
\text { class }\end{array}$ \\
\hline Trinity & $\begin{array}{l}\text { Associate director in } \\
\text { department of } \\
\text { residential living and } \\
\text { learning }\end{array}$ & 2 years & 7 years & $\begin{array}{l}\text { bachelors and } \\
\text { masters }\end{array}$ & $\begin{array}{l}\text { Black/African } \\
\text { American }\end{array}$ & Female & heterosexual & able & $\begin{array}{l}\text { middle } \\
\text { class }\end{array}$ \\
\hline Zuri & $\begin{array}{l}\text { Program } \\
\text { administrator }\end{array}$ & 2 years & $\begin{array}{l}2 \text { to } 3 \\
\text { years }\end{array}$ & $\begin{array}{l}\text { bachelors and } \\
\text { masters }\end{array}$ & Black & Woman & heterosexual & able & $\begin{array}{l}\text { middle } \\
\text { class }\end{array}$ \\
\hline
\end{tabular}

Note. The demographic information in this Table represents how participants self-identified, and we left the language they used even if incorrect or imprecise. For example, some participants identified as "female" for their gender, and we did not change it to "woman." We also acknowledge some discrepancies in the information in the Table. For example, some participants used the language of "heterosexual," and others used "straight;" some used "able-bodied," and others used "able." Some participants also noted the exact amount of time they had been in their position; whereas, some provided the gist amount of time (e.g., "new" or "a few months"). 
Journal Committed to Social Change on Race and Ethnicity | Volume 5, Issue 2 | 2019

For the first phase of the study, we conducted virtual interviews (Google Hangout, Skype) with participants that lasted between 45 and 75 minutes during the months of June, July, and August 2017. One member of the research team conducted each interview using a semi-structured interview format. This format meant we generally asked the same questions, but the semi-structured nature allowed flexibility in followingup on what participants shared or diverting away from the protocol when necessary. We structured the interview into four main parts: Part 1 consisted of rapport-building and questions about learning about participants' racial battle fatigue experiences (e.g., Tell me about a time you experienced racial battle fatigue in your job.). In Part 2, we asked questions about healing strategies for racial battle fatigue (e.g., What strategies do you use to work through racial battle fatigue?). Questions in Part 3 focused on thinking about how other social identities influence participants' experience of racial battle fatigue (e.g., How do your other social identities, like gender and sexual orientation, impact your experience of racial battle fatigue?). Part 4, or the closing set of questions, served to gauge any lingering topics participants wanted to raise that we did not ask them.

All interviews were transcribed verbatim. Three research team members analyzed each transcript; we performed line-by-line coding for the first five transcripts and then met to discuss and compare our notes. In this discussion, given our narrative methodology, we felt uncomfortable breaking up participants' narratives into discrete parts; as such, we modified our analysis approach to use thematic analysis strategies prescribed by Riessman (2008). For the remaining 30 transcripts, three research team members first read the transcript as a whole to get a sense of the story the participant 
Journal Committed to Social Change on Race and Ethnicity | Volume 5, Issue 2 | 2019

was sharing. Following this reading, we used a "chunking" analysis process whereby we treated the transcripts more holistically to identify patterns across the data (Riessman, 2008). We convened after every five transcripts to discuss what stood out to us and reflect on the analysis process. Stephen analyzed all 35 transcripts in order to have a full sense of the data. As we analyzed, we each kept a researcher journal where we wrote in-depth memos about key insights and reflections from the transcripts. At the end of each memo, each research team member wrote a summary of key insights from the transcript. For example, Courtney wrote the following in a memo about Colin.

Colin's interview was the second transcript I analyzed. He, too, situated RBF as this thing that isn't talked about often similarly to Black Panther. Colin spoke to the expectation of POC to do most of the racial justice work, and the toll that takes on someone like him. The double consciousness discussed by DuBois mirrors the level of consciousness student affairs educators of color (specifically Black educators) use to navigate their institutions. However, having colleagues of color on campus to be in solidarity with is helpful when navigating RBF.

Another snippet from a memo comes from the Wilson's researcher journal.

After reading Anna's words, I am wondering about RBF as a condition; something related to a public health issue, i.e., racism as a public health issue and RBF as symptom of that disease. If this is true, then what are we to make of self-care as an antidote? More to the point, as Anna articulates, self-care is a band-aid, not a healing response, thus, how should we take up the work of healing?

Following each research team member writing a memo, we then compared our memos

for discrepancies and similarities. Stephen asked each person to write down their takeaways from the data analysis process. We then shared our takeaways and noted areas of similarity and differences.

\section{Findings}

Our findings illustrate that participants engaged in various strategies for combating racial battle fatigue. Although we initially conflated healing and self-care prior 
Journal Committed to Social Change on Race and Ethnicity | Volume 5, Issue 2 | 2019

to beginning this study, some participants made distinct differences between the two. Participants saw self-care as more temporary fixes to the larger structural issue of racism that enabled them to cope; healing, on the other hand, was more sustainable and attended to their bodies holistically. For this paper, we focus on self-care strategies, and in another paper, we interrogate the ways in which self-care and healing differ for Black student affairs educators (Okello, Quaye, Allen, Carter, \& Karikari, under review). Participants worked to practice self-care from racial battle fatigue in five main ways: (1) unplugging or disconnecting from people and places that made them feel fatigued; (2) building and connecting with community; (3) caring for their bodies; (4) finding safe spaces; and (5) using counseling. Although participants noted overlap in these five ways, we organize the remainder of the findings according to these themes to portray them as distinct strategies.

\section{Unplugging from People and Places}

Participants employed strategies for self-care by unplugging and disconnecting from the people and places that caused them harm and trauma. This also meant unplugging from social media as a strategy for navigating racial battle fatigue. When asked how he practiced self-care, Colin, an assistant dean, stated, "Just unplugging from the world when I get home from work, from my phone, from friends and just kind of re-centering myself." Associate director Trinity said, "Just being able to disconnect and unplug, that's been important. I've just had my time by myself, unplugging, just sitting in the dark, just being able to do that. That's been helpful for me." Both Colin and Trinity noted the importance of unplugging to practice caring for themselves in the midst of racial battle fatigue. 
Journal Committed to Social Change on Race and Ethnicity | Volume 5, Issue 2 | 2019

Imani, a director who has worked in higher education for 15 years, also described unplugging as one of her current ways of coping:

I would say I definitely will just disconnect from social media. I will try to occupy my mind with other things, so that may be me sitting on my couch and watching animated things that are just fun, just so I don't have to think between the lines of what they're saying or try to figure out "Did you mean this?"

Used to having to critique problematic or racist actions, Imani affirmed the importance of watching mindless shows that did not require constant critique and allowed her brain to rest. Similarly, Nicole, a director with 14 years of experience, used TV as a way to unplug: "In my everyday life because I can be rather busy, I like to just veg out, watch TV, sleep, and not think about stuff and actually disconnect." The "stuff" to which Nicole referred is the racism and sexism she experiences. Even if participants knew that unplugging helped with their care, some struggled with doing so. Colin's story is one such example.

Unplugging, that's one I have struggled with because, as a person of color, we always are told, "You have to work two times harder than the average white person to get to where they are," so, I'm often always connected to my email. Even if I go on vacation, I'm often connected, still responding, and I've had to be very intentional of just putting my phone away, turning my email off when I do go away and just focusing on being in the moment whether I'm with my friends or with family.

Colin's comment about needing to work twice as hard is reminiscent of feelings many Black participants hold, which makes taking time away difficult for fear of their white colleagues judging them for not working hard enough. This fear, then, impacted their self-care, which made it difficult to take a break even when on vacation.

Building on Colin's remarks, other participants talked about taking a break or vacation in order to recuperate. "I got to take a break sometimes. I gotta take some days off," Sara, another director, said, "or take a vacation. I need to relax and release, 
Journal Committed to Social Change on Race and Ethnicity | Volume 5, Issue 2 | 2019

and then l'Il come back to this chaotic place." Noting her office as "chaotic," Sara stressed the importance of taking time away from her workplace. Assistant director Joy also mentioned the importance of time away:

I also do not hesitate to just drop everything and leave because sometimes, you just need to. Sometimes, just being in the environment in itself can be very stressful and tiring, and I have no problem just dropping it and leaving, taking a step back and then getting back to it.

Physically leaving their places of work facilitated self-care from racial battle fatigue among participants in the present study.

Some readers might view some of the unplugging strategies noted above as participants avoiding dealing with their racial battle fatigue and as unsustainable strategies. One participant, Mercy, an assistant director with seven years of experience in higher education, in particular, used the word "avoidance" to describe her self-care approach:

It [racism] is too much, and unfortunately, one of the ways that I cope is to just try to put blinders on. For me, a lot of it is avoidance. The news stresses me out. I cannot watch the news anymore. I cannot be around white people. There is a lot of power in just taking a break.

Needing to avoid the news, and sometimes even white people, Mercy discussed the importance of avoidance as a strategy for self-care. As researchers, we were struck with this language of "avoidance" and wrestled with our views on this strategy. Without demonizing the self-care strategy of avoidance practiced by some participants, being able to briefly avoid places, activities, and people that caused harm and trauma provided space for self-care. As Trinity advised, "Learn how to find ways to cope and be unapologetic about that." Being unapologetic about avoidance as a strategy assists these educators in detaching and better re-attaching themselves when navigating racial 
Journal Committed to Social Change on Race and Ethnicity | Volume 5, Issue 2 | 2019

battle fatigue rather than allowing themselves to become numb to the insidious nature of racism in and beyond higher education. Disconnecting and unplugging was an approach to self-care in the midst of racial battle fatigue because as the participants described, it enabled them to remove themselves physically, emotionally, and mentally from the racial battle fatigue they were experiencing.

\section{Building and Connecting with Community}

Conversely to unplugging and disconnecting from people and places, participants conveyed how a connection to community assisted in self-care during racial battle fatigue. One effect of racial battle fatigue, participants noted, was feeling isolated. Being able to identify strong community connections helped participants cope in the midst of racism. Participants shared accounts of how building and connecting to a community of people helped circumvent some of the feelings of isolation. Discussing his connection with other Scholars of Color, Black Panther, a coordinator and adjunct instructor who had been in his role for eight years, said, "In talking with them, there was also this sense of solidarity ... I'm not by myself." Moreover, Destiny, Imani, and Jessie Thomas discussed the importance of building community with white "allies and advocates" in spaces where racial battle fatigue was more pronounced. Furthermore, building connections outside of higher education also helped participants navigate racial battle fatigue. For example, participants bell and Anna found connections in a significant other and spirituality, respectively.

Some Black women in the study formed sister circles as a way to navigate racial battle fatigue. For example, Imani said,

Around the time of the Mike Brown shooting, the students wanted to have some type of a forum to discuss the things that were going on. I had a sister circle, and 
Journal Committed to Social Change on Race and Ethnicity | Volume 5, Issue 2 | 2019

it was a circle of women, Black staff on the campus, who would go to lunch once a month to just have that space to just be ourselves.

Lo, an associate director, offered a similar strategy:

There's some Black girls that work across campus, we go and have lunch every now and then. Those [lunch meetings] are like mini-retreats to get you to a place where you talk in solidarity with some people that you connect with because it's just not always organic with the people that you work with.

Imani and Lo needed community with other Black women in order to navigate racial battle fatigue. This strategy provided them with space to "just be ourselves" and find solidarity with people with whom they connect. Eugene, an assistant director with five years of experience, engaged in a similar approach with Black men student affairs educators on his campus:

I think that's fatigue for staff members, being there to support students but still trying to advocate to them for the administration and just the toll it takes on your soul. What I went ahead and did was put together a lunch meeting for all of the Black professionals on campus, very specifically, the men. I think there's value in bringing the men together, and so I bought them together, and that's been a great just sounding board.

Thus far, we have shared examples of participants finding community within their workspaces. However, participants also noted the importance of connecting with people outside of work or connecting with colleagues beyond the work setting. Joy and Trinity, an assistant director and associate director respectively, provided two such examples:

I went about getting to know other people in my age group around campus and doing things with them on the weekends, just putting myself out there, and so I would be doing things that I wouldn't necessarily do on the usual, but doing that just to get my mind off of work. I like to have people in different departments so that we're not always talking about the students, talking about this incident, talking about this policy that we need to pass. Being with people who don't have the same job, that's helped a lot. - Joy

I've been really good about talking about things with people who aren't in higher ed. I have good friends who are in all types of professions, so I literally can talk to 
Journal Committed to Social Change on Race and Ethnicity | Volume 5, Issue 2 | 2019

them about how I am feeling and go through all of that and get it off my chest and have somebody who are like, "How can I best address this?" - Trinity

Developing community beyond their department enabled Trinity and Joy to care in the midst of racism. Having people outside of work with whom to share ideas and ask questions provided a respite from the day-to-day challenges they faced on campus.

One of the most pernicious effects of racism is how isolating it makes one feel. Being able to share their experiences with others lessened this isolation, one of the benefits of community. Maria, an academic advisor who had only been in her position for four months, attested to this feeling: "The best way to feel affirmations [is through] hearing other people's experiences, especially from other women of color or other

people of color, period. And for us to share our experiences and just so you know you're not alone." For Ray, a coordinator, not keeping his isolation feelings inside was helpful: "I have conversations with friends and vent to them about things going on instead of keeping it bottled up." Both of these participants expressed the importance of connecting with other people of color in community in order to navigate their racial battle fatigue.

Whether it is Black women participants developing solidarity with other Black women who experience the effects of racism and sexism simultaneously; identifying white allies and advocates; or developing a more intimate connection, community was another way in which the Black student affairs educators worked to self-care together to navigate racial battle fatigue.

\section{Caring for One's Body}

We found that participants took care of their bodies physically and mentally as a strategy to self-care in the midst of racial battle fatigue. Engaging in physical activity 
Journal Committed to Social Change on Race and Ethnicity | Volume 5, Issue 2 | 2019

assisted participants in dealing with racism. A coordinator, Elaine, explained how being physical enabled her to manage the energy around racial battle fatigue: "As far as trying to regroup and recharge, l'll go work out at the gym to get some of that excess energy, or that negative energy, and I'll go kick my workout's butt." Like, Elaine, bell, also a coordinator, mentioned being physically active: "I also started running because running helps me clear my mind." When a research team member asked Colin what strategies he used to combat racial battle fatigue, he stressed the importance of work/life balance by sharing how he managed the stress at work by taking care of his physical health:

Working out has been something that's been really good. I take a cycling class every week and go to the gym probably almost every day just to kind of center myself and just to process my thoughts. I also have gotten into doing massages and taking care of myself physically because I have seen the way that stress has impacted my health, and so that's become very important for me.

Being physically active for Colin and bell had an associated positive mental health benefit as well. Sometimes, participants recharged from a walk around campus, as Destiny highlighted:

I have a Fitbit. That's kind of how I recover. There's sometimes I make myself take a lunch, which I often don't do, and I'll just go and walk a few laps around campus for that hour just so I can get out of my office and just get away from all that's coming at me.

For some participants, physical activity had positive effects on their minds as well. Black Panther conveyed this point: "I started doing breathing methods. My mind would race, and it wouldn't stop racing until either I sit there and figure out or I severely occupy myself in something that is very active." When asked what she needs to continue navigating racial battle fatigue, Anna May, a new student affairs educator with one year of experience, responded, "It's a lot of things, like prioritizing my physical health. Because physical health goes hand in hand with my mental and my emotional 
Journal Committed to Social Change on Race and Ethnicity | Volume 5, Issue 2 | 2019

health, and like what am I putting in my body." Anna and Black Panther stressed the link between their physical health and mental health.

Two participants, in particular, used the language of "mindfulness" to illustrate how taking care of their bodies mattered. For example, Elaine noted, "I try to do a lot of mindfulness activities just so I can remain grounded because I know if I don't do it, then nobody else is gonna do it." Relatedly, associate director Kate said, "I set time aside to really learn some mindfulness practices and learn how to be more kind to myself. Some of that now looks like meditation and mindfulness or just deep breathing." Practicing kindness in the midst of racial battle fatigue, Kate voiced, was a strategy that fostered care for herself.

Expanding on the notion of the importance of mental health, Jessie Thomas, with 12 years of experience in higher education, underscored prioritizing activities that enable one to remain engaged mentally. "Whatever self-care means to you, if it's going to a yoga class, if it's cycling, if it's walking outside, you must do that in order to remain healthy and to remain engaged. If not, it [racism] becomes overwhelming." Later in the interview, he stated, "Take care of yourself first. A lot of people call them mental health days, take those mental health days." Mental health was something important to Jessie Thomas, and he indicated the necessity of taking care of oneself through mental health days. Similarly, Joy, an assistant director, commented on how the day after the 2016 presidential election warranted her need for a mental health day: "That was a very tiring day. That was a day I had to call in sick, and I wasn't even sick, but I was mentally drained. I was down, and I just needed to do nothing." Calling in sick in order to have 
Journal Committed to Social Change on Race and Ethnicity | Volume 5, Issue 2 | 2019

time away from their workspaces provided participants with the respite they needed to care for their bodies.

Finally, Ray offered a particularly intriguing strategy to care for himself physically and mentally:

Mondays and Fridays, I block out my calendar for my opportunities to focus on what's going to make me operate at $100 \%$ throughout the week. I keep that time to myself so l'm not in meeting or l'm not starting my week on a terrible note and ending my week on a terrible note. I start my week and end my week on a high regardless, because that time is for me.

Prioritizing time for himself enabled Ray to start and conclude his week "on a high," knowing he needed that high to function at full capacity and bring his best self to work in the midst of facing racism. In summary, the aforementioned quotations illustrate ways participants took care of their bodies both physically and mentally in order to navigate the racial battle fatigue they experienced in their work environments.

\section{Finding Safe Spaces}

Another strategy participants used to self-care was finding spaces whether on or off-campus that were safe to be their whole selves. The previous strategy of community involved connecting with people, while safe spaces refers to the physical spaces participants used to care for themselves. Imani described safe spaces in this way: "Find that space where you are able to be your authentic self and create a space where you all can discuss what you're going through." Colin's personal office was such a space: "When I walk in to my personal office, is often my safe haven and the place that rejuvenates me."

Finding such a safe space, however, was not always possible, as Mercy indicated. "Where can I go? Where is safe? Where can I feel safe? Where can I go 
Journal Committed to Social Change on Race and Ethnicity | Volume 5, Issue 2 | 2019

where l'll be understood, you know, where my actions won't be misinterpreted or I won't be misidentified? Just where is that?" Even when participants found these spaces, sometimes they reinforced oppression of other subordinated identities. Jeff, an assistant professor, pointed out that just because Black people experience racism does not mean they, at times, do not also oppress Black people who hold other minoritized identities:

We need to create a healing space, but we create a healing space around one particular identity or set of identity-related issues, and it comes with microaggressions, or other slight tensions, whatever, from other intersecting identities. Like, "Oh, we're gonna create this healing space for Black folks, but it" terribly misogynistic, you know?" Or, maybe it's not terribly misogynistic, but there are notes of misogyny and anti-LGBTQ sentiments in that space.

Consequently, even safe racial spaces were not safe for Black people with other minoritized identities. Centering one identity -- race -- sometimes fostered environments where Black student affairs educators did not feel valued in their other identities beyond race, thereby perpetuating oppression in spaces designed to foster care.

We asked participants to describe where their racial battle fatigue feels most pronounced; several participants answered that their office is the place they feel most fatigued. For example, Nicole, a director, said, "When I'm not in my office, I feel lighter." When asked what was it about her office space that felt so heavy, she remarked, "I feel like I'm representing for my people. It's a big deal to be a Black woman in leadership." Feeling the need to represent her "people" (i.e., Black women), Nicole indicated, "I actually feel a lot lighter outside of my office." Sedgwick, a director who had been in the role for one-and-a-half years, joined a virtual safe space because he did not have physical safe spaces on his campus:

I just go in that group and say, "[l'm] having a rough day, just pray for me," and everybody's, "We got you, we're praying for you, we got you." Or even just advice, too, so, "Here's a situation, here's what happening with the students, 
Journal Committed to Social Change on Race and Ethnicity | Volume 5, Issue 2 | 2019

here's what's happening with me, what have you all done?" And the level of professionals in that group, it ranges, again, from graduate school up to 10,15, 20 years in the field, so you get a wide array of advice, understanding that it's not a $100 \%$ safe space. You still have to be careful what you're saying, but for me, it's a little more safe than my current environment.

The virtual space Sedgwick described is the Black Student Affairs Professionals Facebook group, where he went for support navigating racism and the resulting racial battle fatigue. Although Sedgwick noted that the space was "not a 100\% safe space," he experienced it as safer than his work environment, and the space provided an immediate support group.

Places to feel safe and free to be their whole selves were important in participants' self-care. For some Black student affairs educators, they found these spaces on their campus, and others resorted to the Internet to find such a space. Even when participants had safe spaces on their campus, sometimes colleagues in these spaces perpetuated oppression of other minoritized identities. Safety, therefore, seemed fleeting and yet was necessary for self-care in racial battle fatigue.

\section{Using Counseling}

A significant strategy that Black student affairs educators in the present study utilized to practice self-care was therapy. Counselors provided participants with a trained professional with whom to talk and seek resources for navigating racial battle fatigue. Although counselors did not solve the racial battle fatigue participants felt, therapists provided needed space to reflect and feel heard in the process. Nathan, a student affairs educator with eight years of experience, was quite enthusiastic about his mental health practices, including counseling:

I make sure that I'm exercising, I make sure that I'm talking about what's bothering me, writing about what's bothering me, and that I'm meeting with 
Journal Committed to Social Change on Race and Ethnicity | Volume 5, Issue 2 | 2019

professionals to make sure l'm taking care of my health. I need my mental health, so l've always used the counseling system.

Like Nathan, Mercy advocated fiercely for counseling when she noted, "I also see a therapist regularly, which to be quite honest, has been most helpful. The therapy thing has been working for me." As researchers, we were pleased to see several participants talk so openly about counseling as a strategy for navigating racial battle fatigue.

When asked what advice she would give to a Black student affairs educator who is experiencing racial battle fatigue, Destiny answered,

Taking care of yourself and not to feel bad about that. My staff knows, and even my boss knows, that when it's 3:30, I'm out of here. I'm going to therapy. And I'm honest, I say, "I'm going to therapy." And I tell my students, "I'm going there because I need to work some stuff out. And I will be back."

Normalizing therapy was a strategy Destiny used to self-care in the midst of racism.

Communicating to her supervisor and students that 3:30 was her time to go to therapy validated the importance of it as a strategy for navigating racial battle fatigue. Similarly, Elaine said,

We had a counselor that was contracted the students during the sit-in. And I was able to talk to her about what was happening and how it was affecting my mental health. Even though I knew I had support, it still felt like it wasn't enough. Don't be afraid to go and find a counselor who's willing to talk to you or just listen to what you have say. Because I think that helped a lot.

Like Destiny, Elaine underscored the importance of counseling as a strategy for working with her mental health. Despite having a good support system, Elaine still needed a trained professional who could listen to her experiences navigating racial battle fatigue. bell also added,

I started going to counseling. My counselor is this Black man who specializes in racial identity development. Being in his space was very familial ... If I wasn't coming here, I would've been a wreck because it [racism] toyed with my emotions. 
Journal Committed to Social Change on Race and Ethnicity | Volume 5, Issue 2 | 2019

The comments from bell illustrate the importance of participants not simply going to counseling, but finding counselors with whom they can relate and who specialize in understanding how racism manifests. All three of the aforementioned participants highlighted the importance of counseling for working with their racial battle fatigue.

Although participants believed in the utility of counseling for students with whom they worked, some had trouble prioritizing counseling for themselves or taking the time to see a therapist. Colin shared one such example:

In terms of going to counseling, we often refer our students to do [counseling]. [We say,] "It'll help you with your situation," but as professionals, it's not something that we always do. This year, one of my goals for myself is I really wanted to practice self-care and to go talk to somebody about some of the situation l've been through and things that l've gone through and just to be able to get it out. I think that it's good to vent to your peers but having somebody that you can just dump it on is very helpful.

Knowing the importance of counseling for students, Colin struggled to embrace counseling for himself in the past. "I was going to go get counseling," Black Panther said, "but I never did." When asked how she is working to heal from racial battle fatigue, Maria replied, “I couldn't give an explanation of the best way I have dealt with it because I don't think I have been dealing with it. I haven't talked to any professional counselor or anything like that." Although we are unsure of why Colin and Maria did not seek counseling, their comments are consistent with some other participants in the present study. Some were forthright about going to counseling and valuing its helpfulness, while others saw its importance but did not commit to going to see a therapist. If participants believed in a stigma attached to Black people seeking counseling, they did not verbalize such a stigma. 
Journal Committed to Social Change on Race and Ethnicity | Volume 5, Issue 2 | 2019

\section{Discussion and Implications for Practice}

In this study, we centered Black student affairs educators' narratives in a society where anti-Blackness is rampant in order to prioritize the voices and needs of participants and validate that their experiences are real and worthy of highlighting. Because racism is so deeply entrenched into the fabric of United States society, surviving in one's Black body necessitates developing healthy ways to cope with racism. Participants in the present study navigated racism through practicing self-care.

Participants engaged in self-care through unplugging from social media and people and places that caused them harm, connecting with community, caring for their bodies holistically, finding and creating safe spaces, and seeking therapy. Many participants engaged in several or all of these strategies and moved in and out of various approaches depending on the situation and their needs. Readers might notice that the aforementioned strategies are fairly common self-care approaches. What makes their use unique by participants in the present study is that they did not subscribe to the notion that they did not have time to prioritize their needs or were selfish for doing so. Black student affairs educators, particularly those who identify as women, sometimes believe they cannot pause to center their own needs (Clark, 2019). In fact, Black women student affairs educators often engage in helping others at greater rates than their Black men counterparts (Howard-Hamilton, Palmer, Johnson, \& Kicklighter, 1998). As such, practicing self-care resisted the notion that caring for their needs was not possible or important.

Worth noting is how self-care strategies enabled participants to cope in the midst of racism. Their strategies did not prevent them from being exhausted or developing 
Journal Committed to Social Change on Race and Ethnicity | Volume 5, Issue 2 | 2019

negative health implications from racism; rather, these strategies enabled them to survive in the midst of racism. After writing these findings, we wondered: is true healing from racism ever possible when racism is such a ubiquitous, everyday experience? What does true healing look like? In another paper (Okello et al., under review), we address these questions around healing, but we raise the questions here given what Arnold et al. (2016) found about excessive coping among Black educators. Although the participants in this study demonstrated resilience in the midst of racism, we want to push back on this notion of resilience or grit (Duckworth \& Duckworth, 2016).

Celebrating and applauding Black student affairs educators for developing strategies to survive racist white environments is important, and yet, when the message of surviving and celebrating that survival continues to be perpetuated, white student affairs educators (and even Black student affairs educators) may come to accept systemic racism and expect Black educators to merely cope with racism.

McGee and Stovall (2015) underscore how taxing it is for Black people to manage race-related stress constantly. How might participants' white colleagues take the onus for redressing racist systems so that Black student affairs educators do not have to develop coping strategies to survive racism? Imagine if participants in the present study could, instead, expend energy into more life-affirming, creative ways that are not tied to developing ingenuity to cope with racist campuses.

White student affairs educators often place the onus on Black educators experiencing racial battle fatigue to practice self-care even though Black people are not responsible for racism. We often wondered how these self-care strategies were addressing the root of racism—white supremacy. When educators do not address 
Journal Committed to Social Change on Race and Ethnicity | Volume 5, Issue 2 | 2019

racism systemically, the strategies in which participants engaged might be band-aids. We want to stress that we are not blaming participants for their self-care strategies or denigrating them; rather, we are putting the responsibility on their white colleagues for addressing systemic racism.

Although self-care presumes an individual process that one person uses to take care of themselves, participants in the present study illustrated how community was important in helping them navigate racial battle fatigue. Sambile (2018) stressed the urgency to move from self-care to community-care in student affairs, so that student affairs educators of color are not charged with the sole responsibility of devoting the time and generating the resources necessary for their own care. Practicing self-care in community shifts from an individual responsibility to cope to providing a sense of solidarity with other educators who also are experiencing racial battle fatigue. This community-care practice helped participants to not feel so isolated in the midst of racial battle fatigue.

The importance of community was also manifested in the virtual spaces Black student affairs educators created because they sometimes did not have other Black colleagues with whom to connect on their historically white campuses. Nicolazzo (2017) found that virtual spaces enabled transgender people to find community with others, and Miller (2017) found the same for disabled queer youth. At the risk of co-opting this work and applying it to Black educators in the study, they, too, talked about the significance of the Black Student Affairs Professionals Facebook group. However, even when participants created spaces to affirm their Black racial identities, members in 
Journal Committed to Social Change on Race and Ethnicity | Volume 5, Issue 2 | 2019

these spaces sometimes reinforced misogynistic, queer- and transphobic messages, complicating the seemingly "always helpful" nature of safe spaces.

Finally, normalizing therapy was a strategy in which participants engaged to work through racial battle fatigue. Often a stigma is attached to Black people seeking counseling, and counselors have not always been helpful to Black people or have known how to validate their experiences with racism (Hyun, Quinn, Madon, \& Lustig, 2006). The fact that so many of the participants in the present study talked so freely about their own therapy helps validate its importance and pushes back against a possible narrative that counseling is not for Black people. One participant in particular illustrated the importance of finding counselors who center race and with whom one can connect, which is often difficult to find, especially in historically white environments.

Given the pervasiveness of racism, Black people must find ways to care for themselves to navigate their racial battle fatigue. This study offers readers strategies they might also use themselves to combat the isolation they might be feeling; we also offer readers insight into the depth of racial battle fatigue and how they might support their Black colleagues in carving out time for self-care. Although the concept of racial battle fatigue is not new, our study provides insight into how Black student affairs educators work to care for themselves in the midst of this fatigue. 
Journal Committed to Social Change on Race and Ethnicity | Volume 5, Issue 2 | 2019

\section{References}

Alexander, E. (2004). The black interior: Essays. New York, NY: Graywolf Press. Arnold, N. W., Crawford, E. R., \& Khalifa, M. (2016). Psychological heuristics and faculty of color: Racial battle fatigue and tenure/promotion. The Journal of Higher Education, 87(6), 890-919.

Bell, D. A. (1992). Racial realism. Connecticut Law Review, 24(2), 363-379.

Beauboeuf-Lafontant, T. (2008). Listening past the lies that make us sick: A voicecentered analysis of strength and depression among black women. Qualitative Sociology, 31(4), 391-406.

Clandinin, D. J., \& Connelly, F. M. (2000). Narrative inquiry: Experience and story in qualitative research. San Francisco, CA: Jossey-Bass.

Clandinin, D. J., \& Rosiek, J. (2007). Mapping a landscape of narrative inquiry: Borderland spaces and tensions. In D. J. Clandinin (Ed.), Handbook of narrative inquiry: Mapping a methodology (pp. 35-75). Thousand Oaks, CA: Sage.

Clark, T. (2019, January 11). This is what Black burnout feels like. Buzzfeed News. Retrieved from https://www.buzzfeednews.com/article/tianaclarkpoet/millennialburnout-black-women-self-care-anxiety-depression

Corbin, N. A., Smith, W. A., \& Garcia, J. R. (2018): Trapped between justified anger and being the strong Black woman: Black college women coping with racial battle fatigue at historically and predominantly White institutions. International Journal of Qualitative Studies in Education, DOI: 10.1080/09518398.2018.1468045

Delgado, R., \& Stefancic, J. (2012). Critical race theory: An introduction (2nd ed.). New York, NY: New York University Press.

DiAngelo, R. (2011). White fragility. International Journal of Critical Pedagogy, 3(3), 5470.

Duckworth, A., \& Duckworth, A. (2016). Grit: The power of passion and perseverance (Vol. 124). New York, NY: Scribner.

Duckworth, A. L., Peterson, C., Matthews, M. D., \& Kelly, D. R. (2007). Grit: Perseverance and passion for long-term goals. Journal of Personality and Social Psychology, 92(6), 1087. 
Journal Committed to Social Change on Race and Ethnicity | Volume 5, Issue 2 | 2019

Dumas, M. J., \& ross, k. m. (2016). "Be real Black for me": Imagining BlackCrit in education. Urban Education, 51(4), 415-442.

Dweck, C. S. (2006). Mindset: The new psychology of success. New York, NY: Ballantine Books.

Evans, L., \& Moore, W. L. (2015). Impossible burdens: White institutions, emotional labor, and micro-resistance. Social Problems, 62, 439-454.

Husband, M. (2016). Racial battle fatigue and the Black student affairs professional in the era of\# BlackLivesMatter. The Vermont Connection, 37(1), 91-98.

Harris-Perry, M. V. (2011). Shame. In Sister citizen: Shame, stereotypes, and Black women in America (pp. 101-133). New Haven, CT: Yale University Press.

Howard-Hamilton, M. F., Palmer, C., Johnson, S., \& Kicklighter, M. (1998). Burnout and related factors: Differences between women and men in student affairs. College Student Affairs Journal, 17(2), 80-91.

Hyun, J. K., Quinn, B. C., Madon, T., \& Lustig, S. (2006). Graduate student mental health: Needs assessment and utilization of counseling services. Journal of College Student Development, 47(3), 247-266.

Kendi, I. X. (2019). How to be an antiracist. New York, NY: One World.

Love, B. L. (2019). We want to do more than survive: Abolitionist teaching and the pursuit of educational freedom. Boston, MA: Beacon Press.

McGee, E. O., \& Stovall, D. (2015). Reimagining critical race theory in education: Mental health, healing, and the pathway to liberatory praxis. Educational Theory, 65(5), 491-511.

McKay, C. (1919). If we must die. The Liberator, 2(6), 1.

Miller, S. A. (2016). Easier said than done: Practicing self-care and health and wellness in higher education and student affairs. The Vermont Connection, 37, 138-144.

Miller, R. A. (2017). "My voice is definitely strongest in online communities": Students using social media for queer and disability identity-making. Journal of College Student Development, 58(4), 509-525.

Nicolazzo, Z. (2017). Trans* in college: Transgender students' strategies for navigating campus life and the institutional politics of inclusion. Sterling, VA: Stylus. 
Journal Committed to Social Change on Race and Ethnicity | Volume 5, Issue 2 | 2019

Okello, W. K., Quaye, S. J., Allen, C. R., Carter, K. D., \& Karikari, S. N. (under review). Racial battle fatigue and the impossibilities of healing. Journal of College Student Development.

Patel, L. (2016). Decolonizing educational research: From ownership to answerability. New York, NY: Routledge.

Pierce, J. L. (1996). Gender trials: Emotional lives in contemporary law firms. Berkeley, CA: University of California Press.

Riessman, C. K. (2008). Narrative methods for the human sciences. Thousand Oaks, CA: Sage.

Sambile, A. F. (2018) Energy exchange: The urgency to move from self-care to community-care in student affairs. The Vermont Connection, 39, 32-39.

Schreiner, L. A. (2010). The "Thriving Quotient": A new vision for student success. About Campus, 15(2), 2-10.

Smith, W. A. (2004). Black faculty coping with racial battle fatigue: The campus racial climate in a post-civil rights era. In D. Cleveland (Ed.), A long way to go: Conversations about race by African American faculty and graduate students (pp. 171-190). Thousand Oaks, CA: Sage.

Smith, W. A. (2009a). "Campus wide climate: Implications for African American students." In L. Tillman (Ed.), A Handbook of African American Education (pp. 297-309). Thousand Oaks, CA: Sage.

Smith, W. A. (2009b.). Higher education: Racial battle fatigue. In R. T. Schaefer (Ed.), Encyclopedia of race, ethnicity, and society (pp. 615-618). Thousand Oaks, CA: Sage.

Smith, W. A., Allen, W. R., \& Danley, L. L. (2007). "Assume the position ... you fit the description": Psychosocial experiences and racial battle fatigue among African American male college students. American Behavioral Scientist, 51(4), 551-578.

Smith, W. A., Hung, M., \& Franklin, J. D. (2011). Racial battle fatigue and the miseducation of Black men: Racial microaggressions, societal problems, and environmental stress. The Journal of Negro Education, 80(1), 63-82. 
Journal Committed to Social Change on Race and Ethnicity | Volume 5, Issue 2 | 2019

Smith, W. A., Mustaffa, J. B., Jones, C. M., Curry, T. J., \& Allen, W. R. (2016): "You make me wanna holler and throw up both my hands!": Campus culture, Black misandric microaggressions, and racial battle fatigue. International Journal of Qualitative Studies in Education, DOI: 10.1080/09518398.2016.1214296

Smith, W. A., Yosso, T. J., \& Solorzáno, D. G. (2006). Challenging racial battle fatigue on historically white campuses: A critical race examination of race-related stress. In C. A. Stanley (Ed.), Faculty of color: Teaching in predominantly white colleges and universities (pp. 299-327). Bolton, MA: Anker.

Squire, D. D., \& Nicolazzo, Z. (2019). Love my naps, but stay woke: The case against self-care. About Campus, 24(2), 4-11.

Sue, D. W. (2010). Microaggressions in everyday life: Race, gender, and sexual orientation. Hoboken, NJ: John Wiley \& Sons, Inc. 